\title{
Chantal Danjou: Dans le dialogue du bleu et du gris
}

\author{
Monique Labidoire \\ Rueil-Malmaison, France
}

$\mathrm{T}$ oute écriture chemine vers une communion, vers une compréhension de soi-même qui conduit à la compréhension de l'autre. Souvent le poète puise sa source dans son environnement propre et les lieux de naissance, les racines, sont souvent déterminants dans l'œuvre en cours. Rimbaud et Charleville, Saint-John Perse et la Guadeloupe, Guillevic et Carnac. Pour Chantal Danjou, ${ }^{1}$ son lieu de naissance, l'Algérie, marque profondément la coloration de son écriture. Dès les premiers textes le bleu apparaît dans sa plénitude et son questionnement. Bleu le ciel, bleue la mer, bleu tout ce qui est aimé, depuis l'eau fœtale jusqu'aux branches des arbres. Bleu cruel pour le souvenir obsédant dans l'exil. Bleu métaphore aussi des sentiments, des émotions et des mythes. L'exil, plus transmission que réalité, va se cristalliser dans une terre de découverte: la poésie.

Si toute poésie mène à un exil, à une séparation d'avec le langage quotidien Chantal Danjou nous montre l'exacerbation de son manque qui nous conduit jusqu'à la lucidité : le poète vit dans une île qui pourrait se nommer solitude.

La ville de sa prime enfance, Alger, est déjà prise comme telle, comme si Alger était elle-même une île et de surcroît exilée de la métropole au milieu des sables et de la mer. Et le poète écrit, dans la réminiscence de souvenirs vécus ou acquis :

\section{Une beauté enfermée \\ Dans la ville insulaire \\ s'exhale longuement \\ sur les balcons ensablés.}

Cette œuvre est marquée par l'exil, la rupture et le langage poétique trouve résonance à cet état de faits. Les premiers poèmes sont en recherche, en projet et on y trouve déjà les thèmes qui vont apparaître plus tard, même si le style, le langage, la mise en oeuvre ne sont pas encore exactement trouvés.

Avec le second recueil de Chantal Danjou, Mythe de Migrale, d'un mot complètement créé par le poète à partir du mot migration, nous sommes dans la quête, une quête d'identité 
mais aussi une quête spirituelle qui amène au questionnement. Ici le poète semble vouloir en finir avec l'obsession d'un rêve qui la maintiendrait prisonnière. "O vie agacée", écritelle. Et dans cet agacement il y a peut-être, parfois, ce bleu obsédant.

Le poète confronte ses vérités au matériau poétique. Le monde, fait de vie et de mort, de croyances et d'incertitudes l'amène à s'interroger sur la relation de l'humain avec le sacré, sur la place du poème dans des sociétés où les techniques et les sciences prédominent, parfois avec nécessité. Mais pour Chantal Danjou le poème est lui aussi nécessité vitale.

Si Eluard nous dit que la terre est bleue comme une orange, Chantal Danjou ajoute à cette terre bleue un écrin lacté. Car si elle est poète, elle est aussi femme et mère et toute sa poésie s'imprègne de cette particularité qui dessine la page de son poème comme une ouverture, une naissance, une mise au monde.

L'abandon, la séparation, l'exil, la solitude, nous les retrouvons dans le recueil Muse au Beau visage penché qui est justement le recueil de la douleur exprimée dans l'intensité et la brièveté du poème. Le premier vers de ce recueil nous dit "Le mot soulève la terre" qui nous rappelle le très beau vers de Guillevic "Le poème nous met au monde".

Le destin reprend au poète ce qu'elle doit mettre au monde, ce qu'elle a déjà mis au monde dans l'attente et la joie. Il lui faut donc construire son matériau poétique pour en extraire une lumière unique qui déclinera un tant soit peu sa souffrance. La nécessité fait jour à une transcendance acérée et le langage poétique peut être ici en partie salvateur car il est lui aussi gestation, création, naissance et don de soi. L'intensité de la douleur dicte l'intensité du poème dans une forme nouvelle pour Chantal Danjou, un poème bref de quelques vers seulement où les blancs de la page font place au silence et au recueillement. En voici quelques exemples

Extraits :

Le mot soulève la terre

mottes régulières bruissantes

et le gel sale le vent la pluie

le bec mordoré de l'herbe.

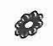

par désir la terre

s'est enveloppée d'un rosier

à petites fleurs jaunes

un souffle chaud autour d'elle 
La glaise repose

sur son flanc droit

elle a l'air las

d'une muse au beau visage penché

La mort retourne la terre

le présent n'a plus de chant

ni de cri l'éphèbe chorégraphique

tremble mouillé de rosée

L'enfant soulève des ombres

dans les champs

voile à l'aube qui enfle et

désincarne l'espoir

Terre respirante

l'enfant en travers

branche morte

des feuilles aveugles sur les yeux.

Le Livre de la soif indique bien le sujet du recueil. Soif des mots, soif de poésie, soif de connaissance, soif de partage et de culture, soif de comprendre le monde principalement à travers et par le poème. Car il semblerait que Chantal Danjou ait découvert une patrie: la patrie poésie. L'exil et la solitude deviennent désormais plus supportables.

Nous trouvons dans ce recueil la nature si présente dans cette poésie mais aussi, la ville, les éléments. Toujours, la nature partage sa création avec la construction humaine, l'une et l'autre s'interfèrent dans le langage poétique et amènent le croisement secret qui délivrera le poème.

On voit apparaître plus précisément ce qui la frappe dans son environnement, dans ses découvertes artistiques et mystiques. Le voyage poétique s'installe et prend appui sur toute circonstance de vie approfondie, sur toute vision immédiate et la réflexion sur l'écriture prend interrogation dans le poème. Ainsi ce vers:

Et glaise aussi du premier verbe travaillé jusqu'à l'eau et aux pierres jumelles. 
Pour le poète, les lieux, les géographies changent, la méditerranée a fait place à l'océan atlantique, la Forêt Noire change ses ténèbres, la plaine dépouille le regard, s'ornemente de vitrail, d'oiseaux, de fleurs et les poèmes commencent à se répondre de recueil en recueil, ainsi ces Halles de fleurs et ces Halles tropicales, dans une soif que seul le poème peut étancher.

Les deux textes conversent d'une zone à l'autre mêlant les civilisations et les cultures, les mots se partagent l'espace, Chine, Acropole, parvis, sirocco, bengali, la cité mélange ses parfums à la nature, cette nature qui seule semble pouvoir donner réponse aux interrogations du poète. Mais le poète, cet éternel aventurier, trouve réponse et Chantal Danjou nous livre une manière d'art poétique intitulée "Le dépouillement":

Le voyageur multiplie ses marches et le poète n'en tire aucune gloire; la peur de son incognito le talonne, la crainte d'une mort sans résurrection, sans parole, sans lumière ni ténèbre derrière lui. L'écriture se nimbe d'un blanc de plus en plus cru, tourbillonne, atomes détachés du verbe initial...Car voilà bien ce qu'il craignait: le blanc absolu, carnivore, érosion de la chair jusqu'à l'os, jusqu'à l'abîme.

Il y a dans cette poésie des mots-thèmes récurrents. L'un d'eux est le mot "concentrique" comme si le poème tournait à la fois dans et hors la sphère, comme si l'écriture "voltigeait" (le mot est du poète) dans et hors le monde, comme si le grand tout vibrait de toutes ses lumières tout en étant en opposition ou en contraste avec l'insularité déjà évoquée.

L'écriture vibre de mille facettes il lui faut tout explorer, ne pas s'arrêter de construire, d'élever, de trouver. Les images explosent dans le verbe, elles nous surprennent, elles cachent derrière leur sens premier des codes secrets que le poète n'est pas toujours décidée à nous livrer afin que l'autre, celui qui lit, l'accueille et fasse lui aussi son chemin. Comme le poète accueille à sa manière les lieux, les couleurs, les mots, les personnes.

Des personnages parcourent les recueils et parmi ces personnages il y en a deux que nous connaissons nous aussi. Il s'agit de Don Quichotte et de Sancho Pansa. Ils apparaissent déjà dans Mythe de Migrale dans une ballade qui leur est dédiée et le poète nous livre ici son interprétation d'un destin qui souligne bien la solitude de deux êtres si différents l'un de l'autre et qui pourtant doivent trouver une force commune pour vaincre.

Le premier texte de Consonnes de sel qui prend pour sujet Don Quichotte est une manière de mise au point, subjective bien sûr, sur le rêve et la réalité, sur la recherche de la lumière, cette connaissance rationnelle et scientifique du monde, sur l'aveuglement qui nous guette tous pour peu qu'il nous arrange. Ainsi Don Quichotte est dans une autre lumière. Il ne peut pas voir les moulins. Il ne peut voir que le poème.

Les Consonnes de sel, accompagnées par Les Mélodies parlées les deux titres du recueil nous donnent une mise en œuvre originale témoignant d'une volonté de passer du discours personnel, sortes de confidences intimistes, à l'écriture plus blanche du poème 
Depuis longtemps, écrit le poète, j'avais envie de joindre journal et écriture poétique en utilisant dans le journal la vision si particulière, si naturellement artificielle de la myope qui ne porte pas ses lunettes. Un angle de vue qui porte à la dérive et à l'excès le paysage et/ou l'être pressenti, qui sait l'implication d'une rencontre-même romancée- sur un lieu....Je souhaite que le lecteur lise en "imbriquant" le journal dans le corps du poème et qu'ainsi son regard puisse se porter alternativement de la page de gauche-écrite en italique- à la page de droite.

Ce que l'auteur ne précise pas c'est que ce n'est pas seulement l'écriture en italique qui change profondément notre vision, c'est le fondement même de la démarche. Nous passons d'une page à l'autre dans l'imbrication de tous les éléments, dans cette volonté de ne pas séparer les arts, la musique, la peinture, l'architecture, la sublimation de la nature ni les sentiments et l'émotion, tout ce qui appartient au sensible, pour parvenir aux "Mélodies parlées" entre deux êtres.Trouver l'équilibre harmonique dans le dialogue, exploit d'autant plus difficile que la volonté du poète c'est d'y inclure la poésie.

Dans ce recueil nous passons de poèmes en prose, ou journal poétique, au poème en vers libres. La mise en œuvre de ces Mélodies parlées nous montrerait que l'exil que nous avions perçu dès les premiers recueils comme une évidence, presqu'une acceptation, serait banni tant l'écriture nous dit maintenant un désir de juxtaposer les différences, de briser la solitude du poème, de le partager mieux, de l'imbriquer, pour reprendre l'expression même du poète dans notre vision quotidienne, dans le déroulement de notre simple parole.

Avec Terres bleues le dernier recueil de Chantal Danjou le dialogue qui s'impose entre le créateur et l'être à venir est de l'ordre de la transcendance mais aussi de l'immanence car le fil du discours murmure juste ce qu'il faut transmettre à l'enfant attendu. Le recueil vit la grande aventure de la naissance, de ces deux naissances simultanées que sont celles de l'enfant et du poème. Il y a peut-être dans cette démarche, une façon de conjurer le mauvais sort qui s'est déjà abattu sur la mère et le poète comme si la parole non seulement pouvait être trace indélébile mais aussi comme si la parole poétique avait don d'éternité.

L'exil est repris ici pour l'enfant à venir qui dès sa venue au monde va quitter le lieu privilégié qui est le ventre de sa mère. Ses racines sont ancrées au profond de l'eau fœtale et sa mémoire inscrira son séjour dans ce lieu de vie, ce même lieu qui a pu être aussi, pour un autre enfant, un lieu de mort, un tombeau.

La mer méditerranée, semble être le seul élément capable d'être mère nourricière. Elles, la mer et la mère, rêvent ensemble le bleu d'enfance, le bleu d'exil, le bleu paradis. Le récit trouve ses marques, indique les seules données qui lui soient essentielles: le questionnement, l'incertitude. 
Nous sommes ivres, écrit le poète, si peu tranquilles sous ce ciel mouvant, et toi la terre fictive, aucune brûlure ne nous enracine.

Les poèmes transmettent la nature, le ciel nu, limpide, les fougères, le sable. Il y a conciliabule entre les lieux et les cultures et aussi entre les habitants de la page.

\author{
Joie de femme \\ si pleine de terre bleue \\ reflétée par l'intensité de l'eau \\ et pénétrée de sa finitude \\ chaque fois qu'elle est mère.
}

et plus loin:

Il n'y a pas de bain possible, seule une macération dans l'utérus bleu de la vague.

Texte et prétexte au langage poétique, texte et prétexte pour le poète d'une interrogation toujours plus vive. L'instant de la mise au monde du poème amène au questionnement de sa source. Plus tard la question pourra se décliner car la tessiture même du texte nous indique à la fois la présence future et l'absence possible.

Ainsi, nous ne pouvons trouver paradoxal que ces "Terres bleues" aient pris inspiration d'un lieu celte, plutôt gris, plutôt pluvieux, dans un pays où les petites maisons s'abritent des grands vents et des tempêtes, où les petits carreaux des fenêtres semblent refuser toute lumière. Le poète nous dit ici des différences qui ne peuvent vivre les unes sans les autres. Les géographies extérieures n'agissent que comme révélateur du monde intérieur du poète. Les alliances créent le langage poétique fait de sens et de forme, de musiques, de cris, d'articulations différentes selon les langues de communication parlées dans tel ou tel pays. Le paysage sculpte les mots autant que les mots sculptent le paysage.

C'est sans doute sur cette démarche que Chantal Danjou s'appuie lorsqu'elle cite en exergue du recueil les mots de Barbara Hepworth qui est sculpteur et dit parlant de son œuvre "Moi le sculpteur, je suis aussi le paysage".

Les terres bleues, si elles idéalisent un sentiment profond de mémoire d'enfance, de climat clément ou de l'esthétique d'un paysage, rejoignant peut-être l'azur mallarméen, nous font prendre conscience de ces différences auxquelles nous sommes quotidiennement confrontés.

Ainsi rien n'oppose le genêt au cactus, rien n'oppose le bleu et le gris, rien n'oppose la ténèbre et la lumière. Tout est union d'amour.

l'univers est bleu ardoise

constellé par les pépites

d'or et de cuivre 
des papillons

et toi la lande

dissoute dans la brume

tu ne laisses de guide

que le parfum du chèvrefeuille

petits éclats de vitrail

les papillons agitent la lumière

jusqu'à la renverser

Et plus loin cette autre parole du poète:

Strates de brume et de lumière alternent: nous avons le privilège de les embrasser toutes dans un regard unique.

La quête d'identité, la quête du graal qui se déclinent peu à peu en quête initiatique trouvent place et se nomment dans la poésie de Chantal Danjou. Dans cette œuvre, il semblerait que la nature puisse donner réponse d'un espace temps renouvelé qui par intuition indiquerait un chemin de vie.

Mais pour les poètes, pour Chantal Danjou, il y aussi l'initiation à l'univers par le poème qui ne serait plus seulement une quête, une attente, mais un espace largement ouvert à toutes lumières.

\section{Note}

${ }^{1}$ Chantal Danjou est née à Alger. Docteur ès lettres, elle collabore comme poète et critique à plusieurs publications françaises et étrangères. Elle a participé à la création de LA ROUE TRAVERSIERE qui se propose de mieux faire connaître les poètes contemporains d'expression française et continue d'animer régulièrement cette association. Elle a publié:

La Cendrifère (1981) chez l'auteur.

Mythe de Migrale (1985), Editions Saint-Germain-des-Prés.

Le livre de la soif (1993), L'Harmattan.

Les consonnes de sel (1995), L'Harmattan.

Muse au beau visage penché (1996), Encres Vives N ${ }^{\circ} 217$

Terres Bleues (1998), L'Harmattan. 